ARTICLE

\title{
Proton gradients from light-harvesting E. coli control DNA assemblies for synthetic cells
}

\author{
Kevin Jahnke ${ }^{1,2}$, Noah Ritzmann ${ }^{3}$, Julius Fichtler ${ }^{1,2}$, Anna Nitschke ${ }^{1,2}$, Yannik Dreher ${ }^{1,2}$, Tobias Abele ${ }^{1,2}$, \\ Götz Hofhaus $^{4}$, Ilia Platzman ${ }^{5,6}$, Rasmus R. Schröder ${ }^{4}$, Daniel J. Müller (1D ${ }^{3}$, Joachim P. Spatz ${ }^{5,6,7}$ \& \\ Kerstin Göpfrich (1D 1,2凶
}

Bottom-up and top-down approaches to synthetic biology each employ distinct methodologies with the common aim to harness living systems. Here, we realize a strategic merger of both approaches to convert light into proton gradients for the actuation of synthetic cellular systems. We genetically engineer E. coli to overexpress the light-driven inward-directed proton pump xenorhodopsin and encapsulate them in artificial cell-sized compartments. Exposing the compartments to light-dark cycles, we reversibly switch the $\mathrm{pH}$ by almost one $\mathrm{pH}$ unit and employ these $\mathrm{pH}$ gradients to trigger the attachment of DNA structures to the compartment periphery. For this purpose, a DNA triplex motif serves as a nanomechanical switch responding to the $\mathrm{pH}$-trigger of the $E$. coli. When DNA origami plates are modified with the $\mathrm{pH}$-sensitive triplex motif, the proton-pumping $\mathrm{E}$. coli can trigger their attachment to giant unilamellar lipid vesicles (GUVs) upon illumination. A DNA cortex is formed upon DNA origami polymerization, which sculpts and deforms the GUVs. We foresee that the combination of bottom-up and top down approaches is an efficient way to engineer synthetic cells.

\footnotetext{
${ }^{1}$ Biophysical Engineering Group, Max Planck Institute for Medical Research, Heidelberg, Germany. ${ }^{2}$ Department of Physics and Astronomy, Heidelberg University, Heidelberg, Germany. ${ }^{3}$ Department of Biosystems Science and Engineering, Eidgenössische Technische Hochschule (ETH) Zurich,

Basel, Switzerland. ${ }^{4}$ Centre for Advanced Materials, Heidelberg, Germany. ${ }^{5}$ Max Planck Institute for Medical Research, Department of Cellular Biophysics, Heidelberg, Germany. ${ }^{6}$ Institute for Molecular Systems Engineering (IMSE), Heidelberg University, Heidelberg, Germany. ${ }^{7}$ Max Planck School Matter to Life, Heidelberg, Germany. ${ }^{凶}$ email: kerstin.goepfrich@mr.mpg.de
} 
S ynthetic biology cultivates an engineering approach to biology with the aim to create or to re-purpose biological parts for specific tasks. The field is commonly divided into two branches with distinct tools and methodologies, but also distinct challenges-top-down and bottom-up synthetic biology ${ }^{1,2}$. The top-down approach uses genetic engineering techniques to manipulate natural cells, reprogramming their behavior and equipping them with unique and exciting functions ${ }^{3}$. Escherichia coli (E. coli) bacteria, for instance, have been engineered for a variety of tasks, including biofuel production $^{4}$, cancer cell targeting ${ }^{5}$ or light harvesting 6,7 . Yet living cells remain too complex to achieve full control and not all added functions are compatible with the host ${ }^{8}$.

The bottom-up approach, on the other hand, has been successful at reconstituting natural biomolecules, or artificial components in cell-sized confinement like microfluidic droplets or lipid vesicles ${ }^{9-11}$. Noteworthy modules have been implemented so far, each mimicking a specific function of a living cell, including energy generation ${ }^{12,13}$, metabolism ${ }^{14}$, motility ${ }^{15,16}$, cytoskeletal contraction $^{17}$ or division ${ }^{18}$. Yet the combination of these modules towards complex signaling pathways for dynamic systems remains challenging. Merging the capacities of top-down and bottom-up approaches to synthetic biology can be a leap forward towards complex bottom-up assemblies but also more versatile and well-defined top-down systems. Leading to this direction, communication between natural and synthetic cells has been implemented ${ }^{19-21}$ and bottom-up assembled vesicles were used as organelle mimics in living cells ${ }^{22}$. Furthermore, engineered prokaryotes have recently been used as artificial organelles in living cells $^{23,24}$, yet this has never been translated into synthetic cells.

Here, we use top-down genetic engineering to equip $E$. coli with light-harvesting capabilities. We employ them as synthetic organelle mimics inside bottom-up assembled synthetic cellular compartments. Thereby, we can reversibly switch the $\mathrm{pH}$ upon illumination to trigger an optical or a mechanical response. The latter is based on the $\mathrm{pH}$-sensitive membrane attachment of a triplex-forming DNA motif triggered by proton gradients from light-harvesting E. coli. Furthermore, we employ the $\mathrm{pH}$-gradients to sculpt synthetic cellular compartments by attaching a DNA origami plate to the $\mathrm{pH}$-sensitive DNA strand.

\section{Results}

Top-down engineering of $\boldsymbol{E}$. coli for light-harvesting. To equip synthetic cells with the capability to generate proton gradients, we set out to assemble an energy module. We genetically engineered E. coli to overexpress the light-driven proton pump xenorhodopsin, a transmembrane protein from nanohalosarchaeon Nanosalina $^{25}$. It contains a retinal which, upon illumination, undergoes a trans-cis conformational change and shuttles a proton across the lipid membrane. We chose xenorhodopsin because it shows unique features compared to other proton pumps, such as bacteriorhodopsin or proteorhodopsin: First of all, xenorhodopsin exhibits a substantially faster photocycle, which can result in larger proton gradients ${ }^{25}$. Second, as an inward-directed pump ${ }^{26}$, xenorhodopsin increases the $\mathrm{pH}$ (instead of decreasing it) in the extracellular space upon illumination (Fig. 1a). As an additional feature, we introduced a Cterminal fluorescent GFP or mCherry tag to xenorhodopsin for vizualization of the E. coli. The choice of two dyes allows us to work with different combinations of fluorophores as required.

To assess and quantify the proton pumping capabilities of the genetically engineered $E$. coli, we performed a photoactivity assay, where we inserted a micro $\mathrm{pH}$ electrode into the $E$. coli suspension and exposed it to multiple light-dark cycles. Since the absorption spectrum of xenorhodopsin covers a broad range, the use of a white light lamp is more effective than excitation with a specific wavelength (Supplementary Fig. 1). Illumination increased the $\mathrm{pH}$ in the extracellular space by almost one $\mathrm{pH}$ unit within five minutes (Fig. 1b), because protons are translocated from the extracellular solution to the cytosol. Longer illumination times resulted in saturation of the $\mathrm{pH}$ change (Supplementary Fig. 2). In the range from $\mathrm{OD}_{600}=8$ to $\mathrm{OD}_{600}=$ 40, the E. coli concentration did not significantly change the obtained $\mathrm{pH}$ gradients and we observed only a very minor increase in the kinetics at higher concentrations (Supplementary Fig. 3). The pH quickly returned to its initial value after the light was turned off due to the dissipation of protons. Even after three complete light-dark cycles, we observed only little decrease in the $\mathrm{pH}$ gradient. Compared to previous reports where proton pumps were reconstituted in lipid vesicles ${ }^{7,27}$, we could achieve faster and higher $\mathrm{pH}$ gradients using genetically engineered $E$. coli. Moreover, the use of $E$. coli circumvented the need for cumbersome protein purification and reconstitution to prepare proteoliposomes $^{28}$, which highlights a key advantage of merging top-down and bottom-up synthetic biology.

As a next step, we aimed to encapsulate the $E$. coli as a $\mathrm{pH}$ switch in synthetic cells, which makes $\mathrm{pH}$ monitoring with an electrode impractical. We thus supplement the E. coli suspension with the ratiometric $\mathrm{pH}$-sensitive fluorescent dye pyranine. The fluorescence properties of pyranine depend on its protonation state (Fig. 1c, Supplementary Fig. 4). After suitable calibration measurements (Supplementary Fig. 5), we could hence monitor the $\mathrm{pH}$ optically ${ }^{29}$. Figure $1 \mathrm{~d}$ plots the fluorescence intensity ratio over time while the system was exposed to light-dark cycles (Supplementary Movie 1, Supplementary Fig. 6). Notably, we obtained the same results as previously with the $\mathrm{pH}$ electrode.

Light-harvesting $\boldsymbol{E}$. coli as internal $\mathrm{pH}$ actuators. Having demonstrated light-activated $\mathrm{pH}$ switching in bulk, we wanted to integrate the engineered $E$. coli as artificial mitochondria mimics in synthetic cell-sized confinements. Using a microfluidic droplet formation device (Fig. 2a), E. coli and pyranine were encapsulated in surfactant-stabilized water-in-oil droplets (Fig. 2b; Supplementary Fig. 7). We obtained E. coli-containing compartments with a radius of $27 \pm 5 \mu \mathrm{m}$ (mean \pm s.d., $\mathrm{n}=53$, Fig. 2c). Pyranine served as a fluorescent $\mathrm{pH}$ indicator inside the compartments (Fig. 2d; Supplementary Fig. 8). We exposed the system to three consecutive light-dark cycles. Illumination with white light triggered a $\mathrm{pH}$ increase inside the cell-sized compartments due to the light-driven proton transport by the E. coli, resulting in an optical response of the compartments themselves (Fig. 2e; Supplementary Movie 2). Taken together, we demonstrated that the genetically engineered $E$. coli can provide light-activated proton gradients in cell-sized compartments.

pH-sensitive attachment of DNA to the compartment periphery. Proton gradients in synthetic systems are especially exciting if they can be utilized to control and energize downstream processes. Instead of relying on purified proteins, an increasingly popular approach is to construct such $\mathrm{pH}$-dependent machineries de novo from molecular building blocks. DNA nanotechnology, in particular, has been employed to build a variety of functional components for synthetic cells ${ }^{17,30,31}$, including membrane-sculpting ${ }^{32-35}$ and $\mathrm{pH}$-responsive components such as filaments ${ }^{36}$ or rotors ${ }^{37,38}$. However, pH-responsive actuation is challenging after encapsulation into a compartment. With the E. coli, we can circumvent this by converting light into a proton gradient.

Towards this goal, we want to implement $\mathrm{pH}$-induced membrane modification and remodeling. For this purpose, we employ a single-stranded DNA sequence, which consists of 
a
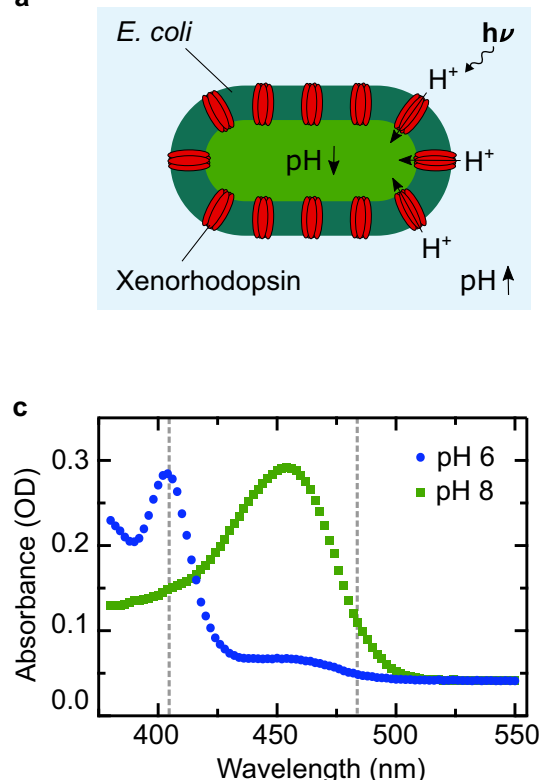

b

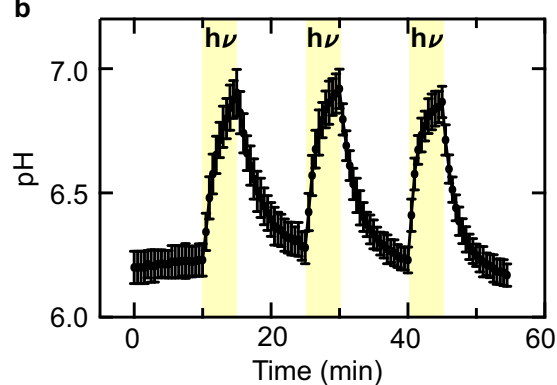

d

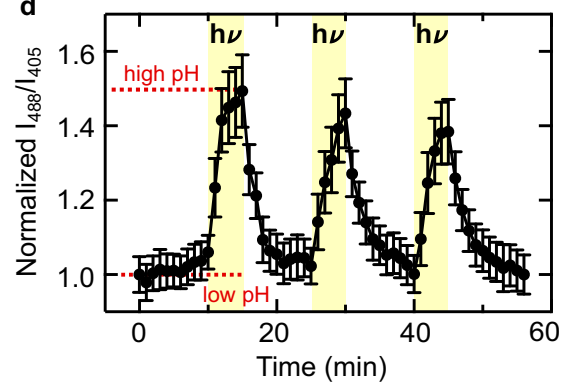

Fig. 1 Genetically engineered xenorhodopsin-expressing $\mathbf{E}$. coli generate a pH gradient upon illumination with white light. a Schematic illustration of an E. coli expressing xenorhodopsin, a light-driven proton pump (red), allowing for the reversible generation of a directional $\mathrm{pH}$ gradient during illumination with white light. The inward pump increases the $\mathrm{pH}$ of the external solution. b Photoactivity generated by the $E$. coli $\left(\mathrm{OD}_{600}=20\right.$, in $\left.150 \mathrm{mM} \mathrm{NaCl}\right)$ measured with an external $\mathrm{pH}$ electrode. The $\mathrm{pH}$ is plotted over time during three light-dark cycles (periods of illumination are indicated in yellow). The $\mathrm{pH}$ increases by almost one $\mathrm{pH}$ unit within $5 \mathrm{~min}$ of illumination and nearly returns to its original value after $10 \mathrm{~min}$ in the dark (mean $\pm \mathrm{s}$.d., $n=3$ ). c Absorbance measurements of the $\mathrm{pH}$-sensitive ratiometric fluorophore pyranine at $\mathrm{pH} 6$ (blue) and pH 8 (green). The $\mathrm{pH}$ can be quantified as the fluorescence intensity ratio at the excitation wavelengths $488 \mathrm{~nm}$ and $405 \mathrm{~nm}$ (gray dashed lines). d Normalized fluorescence intensity ratio $\mathrm{I}_{488} / \mathrm{I}_{405}$ of pyranine $(50 \mu \mathrm{M})$ over time in a solution containing $E$. coli and lipid vesicles as determined with confocal fluorescence microscopy (mean \pm s.d., $n=4)$. Periods of illumination are indicated in yellow. Source data is available for Fig. 1b-d.

specifically designed sections ${ }^{36,39}$ : First, it contains a selfcomplementary section, which forms a DNA duplex following the Watson-Crick basepairing rules. A single-stranded hairpin loop connects the duplex-forming sections. Another critical single-stranded region is located at the $3^{\prime}$ end. At acidic $\mathrm{pH}$ it wraps around the DNA duplex to form a triplex, held together by Hoogsten interactions. At basic $\mathrm{pH}$, the triplex becomes unstable. The remaining duplex can now also open up, if a second DNA strand with higher affinity binds to the hairpin loop ${ }^{36}$. By functionalizing this second DNA strand with a terminal cholesterol tag, it self-assembles at the compartment periphery due to hydrophobic interactions ${ }^{40}$. Thereby, we can recruit the triplex-motif strand to the compartment-periphery in a $\mathrm{pH}$ reversible manner (Fig. 3a). At basic $\mathrm{pH}$, the triplex-motif strand is bound to the periphery (Fig. 3b, inset top right and Supplementary Fig. 9). At acidic pH, on the other hand, it remains homogeneously distributed inside the compartment (Fig. 3b, inset bottom left). Note that the periphery attachment is due to specific interactions between the opened DNA triplex and the complementary cholesterol-tagged DNA. Unspecific absorption in the absence of the cholesterol-tagged DNA was not observed (Supplementary Fig. 10) ${ }^{41}$. To characterize the $\mathrm{pH}$ sensitive membrane attachment, we assessed the fluorescence intensity inside the compartment as a function of $\mathrm{pH}$. The fluorescence intensity decrease with increasing $\mathrm{pH}$ follows a sigmoidal fit with a $\mathrm{pK}_{\mathrm{a}}$ of 6.05 , which is compatible with the $\mathrm{pH}$ range of the E. coli and previous works ${ }^{39}$. It is important to note that the choice of fluorophore can affect the $\mathrm{pH}$ switching point $^{41}$.

As a next step, we need to verify that membrane attachment of the DNA can also be triggered by the engineered E. coli. We hence co-encapsulated them with the cholesterol-tagged as well as the triplex-forming DNA strand using a microfluidic two-inlet device (Supplementary Figs. 7 and 11). A second inlet proved to be advantageous, because the cholesterol-tagged DNA could bind to the droplet periphery before encountering the $E$. coli, hence preventing unwanted attachment to the E. coli due to hydrophobic interactions ${ }^{42}$.

After microfluidic droplet formation in the dark, the triplexforming DNA was homogeneously distributed inside the compartment with some attachment to the periphery (Fig. $3 \mathrm{c}$ ). From the calibration curve, we could deduce a starting $\mathrm{pH}$ value of around 6.2 inside the droplets, consistent with previous experiments in Fig. 1. Upon illumination, the DNA attached to the compartment periphery over the course of $30 \mathrm{~min}$ (Fig. 3d, Supplementary Movie 3). We can deduce a $\mathrm{pH}$ increase of approximately one $\mathrm{pH}$ unit to about $\mathrm{pH} 7.25$, consistent with the bulk experiments in Fig. 1 (Supplementary Note 1). The dynamic opening of the triplex and subsequent attachment to the periphery was considerably slower than the pyranine response ${ }^{36}$. We observed that the DNA remained attached to the compartment periphery after the light was turned off. We found that this is due to an interesting hysteresis effect: Once the DNA duplex at the droplet periphery was formed, the detachment of the triplexforming DNA was shifted to substantially lower $\mathrm{pH}$ values (Supplementary Fig. 12). Therefore, the DNA did not detach when the $\mathrm{pH}$ returned to its original value after turning the light off. This effect can likely be attributed to an effective stabilization of the duplex conformation ${ }^{41}$. Detachment could, however, be achieved with larger $\mathrm{pH}$ gradients (Supplementary Movie 4): Fig. $3 \mathrm{e}$ shows the reversible attachment of the DNA triplex to the compartment periphery, triggered by the addition of a proton acceptor ( 1 vol\% propylamine in HFE) and subsequent addition of a proton donor ( 1 vol\% trifluoroacetic acid (TFA) in HFE). The 
a

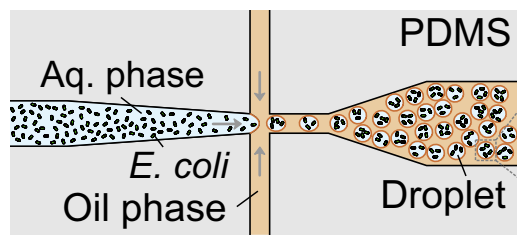

b

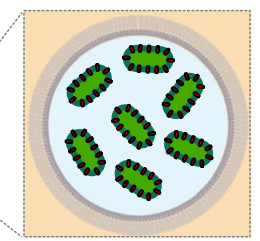

C

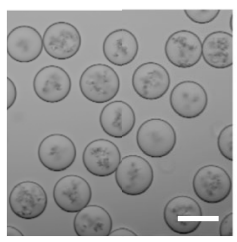

d
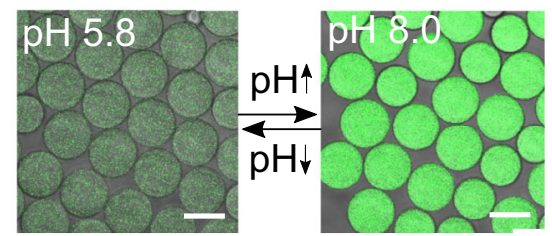

e

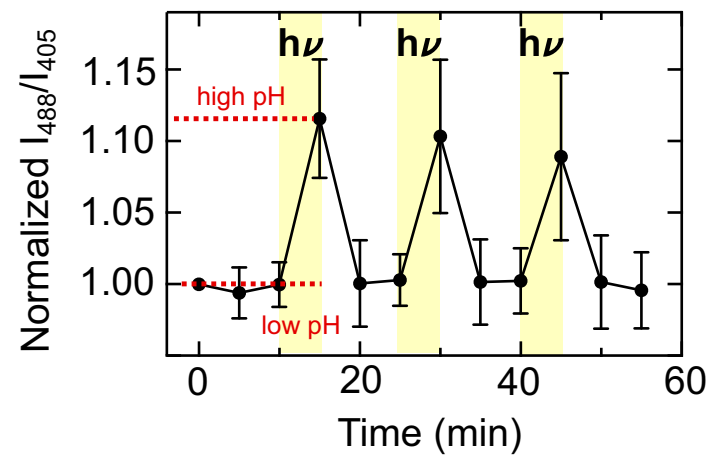

Fig. 2 E. coli as light-activated synthetic organelles that change the pH inside cell-sized confinement. a Schematic illustration of the microfluidic device used to encapsulate engineered $E$. coli and pyranine into cell-sized compartments. Water-in-oil droplets were generated at a flow-focusing T-junction of a PDMS-based device. $\mathbf{b}$ Schematic illustration of a surfactantstabilized water-in-oil droplet containing engineered $E$. coli. c Brightfield image of monodisperse water-in-oil droplets with a radius of $27 \pm 5 \mu \mathrm{m}$ (mean \pm s.d., $n=53$ ) containing engineered $E$. coli $\left(\mathrm{OD}_{600}=20\right)$. Scale bar: $50 \mu \mathrm{m}$. d Overlay of confocal fluorescence and brightfield images of pyranine $\left(\mathrm{c}=50 \mu \mathrm{M}, \lambda_{\mathrm{ex}}=488 \mathrm{~nm}\right)$ inside droplet-based compartments at pH 5.8 and pH 8.0. Scale bar: $50 \mu \mathrm{m}$. e Normalized fluorescence intensity ratio $\mathrm{I}_{488} / \mathrm{I}_{405}$ of $E$. coli and pyranine-containing droplets over time. The fluorescence intensity ratio (mean \pm s.d., $n=11$ droplets) of pyranine (and hence the $\mathrm{pH}$ ) increases reversibly during periods of illumination with white light ( $30 \mathrm{~W}$ halogen bulb, highlighted in yellow). Note that the number of recorded frames was reduced because the illumination light had to be turned off each time an image was acquired, which will bias the proton pumping activity. Source data is available for Fig. 2e.

increase in fluorescence after addition of TFA can be attributed to the $\mathrm{pH}$-sensitive nature of the $\mathrm{Cy} 5 \mathrm{dye}^{43}$.

We have thus realized a complex reaction pathway, where illumination activates the internal organelle mimics, causing a proton gradient which, in turn, leads to the stable modification of the compartment periphery. Moreover, the $\mathrm{pH}$-sensitive membrane attachment and the discovered hysteresis effect extend the scope of the DNA triplex motif in DNA nanotechnology.

pH-induced morphology change. Next, we can exploit the $\mathrm{pH}$ responsive modification of the compartment periphery to provide a meaningful function. Assuming that the DNA triplex motif could serve as a shuttle to bring components to the periphery, we set out to develop a cytoskeleton mimic, which could sculpt synthetic cellular compartments in a $\mathrm{pH}$-responsive manner. For this purpose, we designed a DNA origami plate made of two

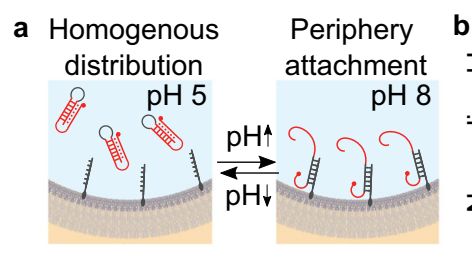

C

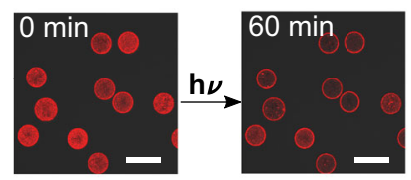

d

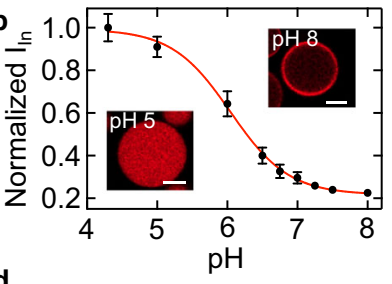

e
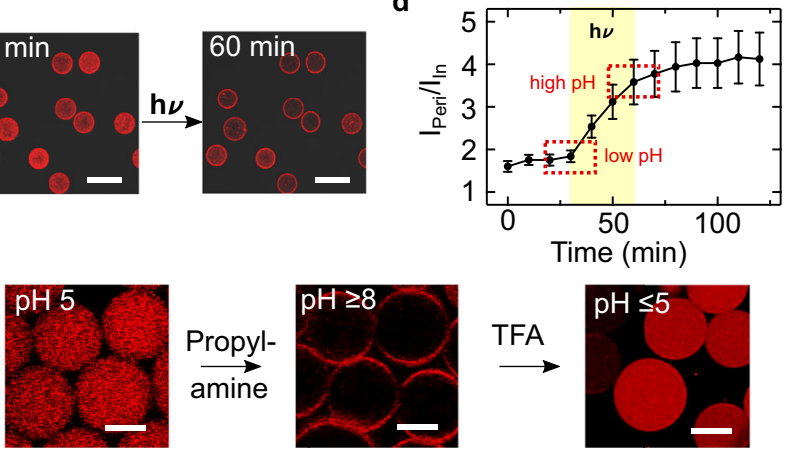

Fig. 3 pH-sensitive DNA attachment to the droplet periphery stimulated with engineered $\boldsymbol{E}$. coli. a Schematic illustration of $\mathrm{pH}$-sensitive duplex formation at the droplet periphery. In response to higher $\mathrm{pH}$, the DNA triplex motif opens up and reversibly attaches to the cholesterol-tagged DNA handles at the compartment periphery. b Normalized fluorescence intensity of triplex-forming DNA inside the droplet (excluding the periphery) dependent on the $\mathrm{pH}$ (mean \pm s.d., $n=20$ ). The sigmoidal fit (red curve) has a turning point at $\mathrm{pH}$ 6.05. The insets depict confocal fluorescence images of Cy5-labeled triplex-forming DNA ( $\lambda_{\text {ex }}=633 \mathrm{~nm}$, $1 \mu \mathrm{M}$ ) inside a water-in-oil droplet (containing $1.5 \mu \mathrm{M}$ cholesterol-tagged DNA) at $\mathrm{pH} 5$ (bottom left) and $\mathrm{pH} 8$ (top right). At pH 8, the triplexforming DNA is located at the droplet periphery, whereas it is homogeneously distributed at pH 5. Scale bars: $20 \mu \mathrm{m}$. c Confocal images of microfluidic water-in-oil droplets containing the triplex-forming DNA $\left(\lambda_{\text {ex }}=633 \mathrm{~nm}\right)$, cholesterol-tagged DNA and engineered $E$. coli before ( $0 \mathrm{~min}$ ) and after (60 min) illumination with white light. Scale bars: $100 \mu \mathrm{m}$. d Fluorescence intensity ratio $I_{\text {peri }} / I_{\text {in }}($ mean \pm s.d., $n=20$ ) of the triplexforming DNA over time. The ratio increases during light illumination due to binding of the triplex-forming DNA to the droplet periphery. The time period of illumination is indicated in yellow. e Confocal images of microfluidic water-in-oil droplets containing the triplex-forming DNA ( $\lambda_{\text {ex }}=$ $633 \mathrm{~nm}$ ) and cholesterol-tagged DNA produced at $\mathrm{pH} 5$ (left image). Flushing of the proton acceptor propylamine ( $1 \mathrm{vol} \%$ in HFE) led to a $\mathrm{pH}$ increase of the aqueous solution inside the droplets and hence attachment of the triplex-forming DNA (middle). Subsequent flushing of the proton donor trifluoroacetic acid (1 vol\% in HFE) decreased the $\mathrm{pH}$ and hence causes DNA detachment (right). The attachment of triplex-forming DNA to the droplet periphery is reversible. Scale bars: $30 \mu \mathrm{m}$. Source data is available for Fig. 3b, d.

layers of DNA helices (Fig. 4a, Supplementary Fig. 13). The two layers were twisted at a $90^{\circ}$ angle as visible in the cryo electron micrographs (Fig. $4 \mathrm{~b}$ ), providing interaction sites for blunt-end stacking ${ }^{44}$ on all four sides of the DNA origami. This, in turn, leads to efficient polymerization of the DNA origami monomers into large flat sheets when the edge staples at the scaffold seam are included as verified via cryo-electron microscopy (Supplementary Fig. 14), atomic force microscopy (Supplementary Fig. 15) and agarose gel electrophoresis (Supplementary Fig. 16). The bottomside of the DNA origami was functionalized with the DNA triplex motif at four positions. At basic $\mathrm{pH}$, the DNA origami thus attached to the periphery of cell-sized droplets functionalized with the complementary cholesterol-tagged strand. However, the droplets remained spherical (Supplementary Fig. 17). This is not surprising given that droplets could also not be deformed with 
a Top:
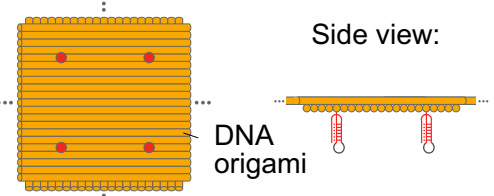

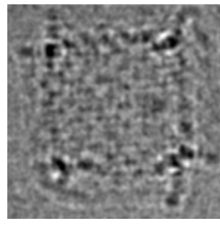

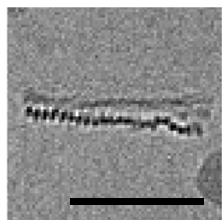

C $\mathrm{pH} 8.3$
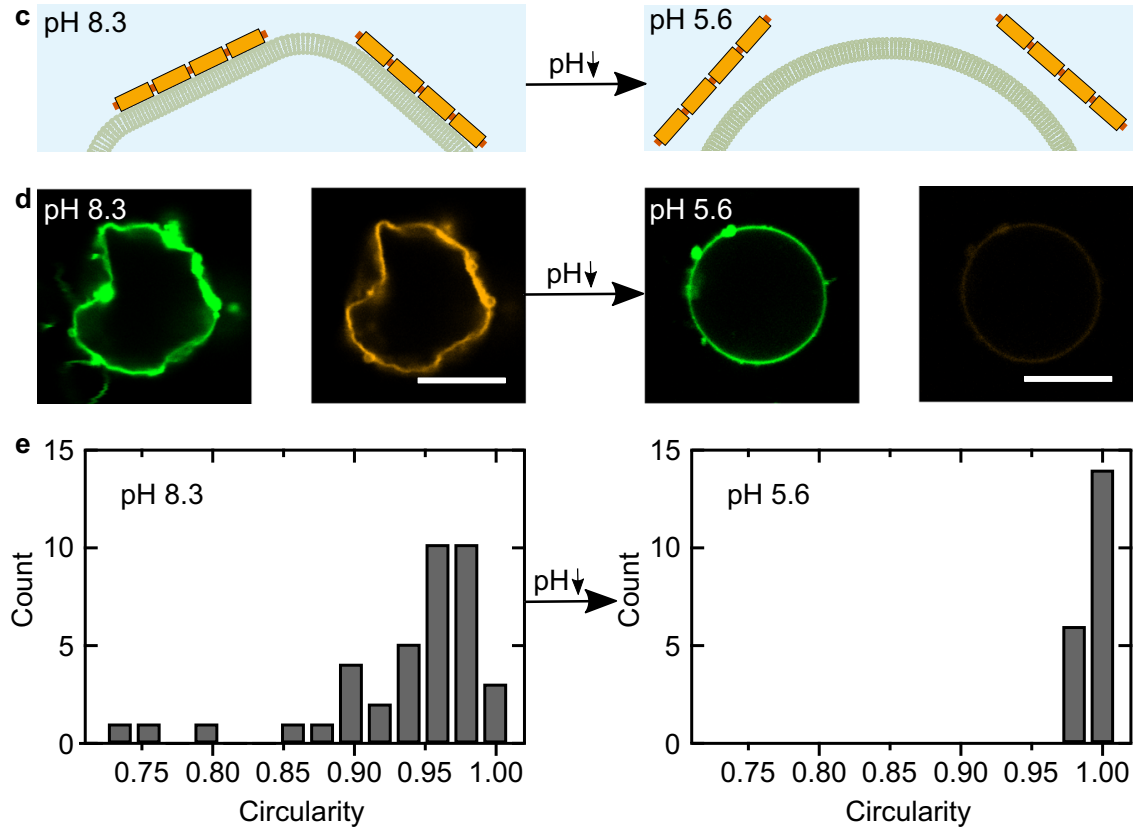

Fig. 4 Deformation of GUVs with pH-sensitive DNA origami. a Schematic illustration of the DNA origami, which can polymerize into flat DNA origami sheets due to blunt end stacking. The DNA origami was functionalized with four DNA triplex motifs (red, two are shown), such that its assembly on the GUV membrane is $\mathrm{pH}$-dependent. b Cryo-EM micrographs of the DNA origami plates. The top view (left) and the side view (right) showing the two DNA layers connected at a $90^{\circ}$ angle. Scale bar: $50 \mathrm{~nm}$. c Schematic illustration of a section of a GUV membrane functionalized with cholesterol-tagged pHsensitive polymerized DNA origami. At high pH the DNA origami sculpts the GUV membrane. At low pH, it detaches and the GUV relaxes into its spherical shape. (Continued on the following page) $\mathbf{d}$ Confocal images of GUVs before (left) and after (right) decreasing the $\mathrm{pH}$ from pH 8.3 to pH 5.6 by addition of iso-osmotic potassium dihydrogenphosphate buffer. The GUV (lipids labeled with Atto $488, \lambda_{\text {ex }}=488 \mathrm{~nm}$ ) is initially deformed due to the membranebound polymerized DNA origami (labeled with $\mathrm{Cy} 3, \lambda_{\mathrm{ex}}=561 \mathrm{~nm}$ ). The DNA origami detaches upon lowering the pH (the fluorescence from the detached DNA origami in the background is too weak to be visible). Scale bars: $10 \mu \mathrm{m}$. e Histograms of GUV circularity before (left) and after (right) lowering the pH. At pH 8.3, the mean circularity is $0.94 \pm 0.06(n=39)$ compared to $0.991 \pm 0.004(n=20)$ at pH 5.6, respectively. Source data is available for Fig. 4 e.

cytoskeletal proteins due to their interfacial properties ${ }^{17,45}$. We thus moved to a compartment system which better mimics the mechanical properties of cellular membranes. We produced giant unilamellar lipid vesicles (GUVs) and functionalized them externally with the cholesterol-tagged DNA. We then added the $\mathrm{pH}$-sensitive DNA origami to the GUVs at $\mathrm{pH}$ 8.3. At this $\mathrm{pH}$, the DNA origami binds to the GUV membrane. Upon addition of the staples at the scaffold seam, which enable blunt-end stacking, we observed considerable deviations from the initially spherical shape of the GUV (Fig. 4c, d). Large flat sections appeared on the GUV with kinks at the phase boundaries between the polymerized flat DNA sheets. In fluorescence recovery after photobleaching (FRAP) experiments, we find that the polymerized DNA origami layer is not diffusive, as expected for large interconnected sheets, in particular in the presence of $\mathrm{Mg}^{2+46}$. For the underlying deformed lipid membrane, we obtain a diffusion coefficient of $1.23 \pm 0.14 \mu \mathrm{m}^{2} \mathrm{~s}^{-1}$ which is comparable to the lipid diffusion in bare GUVs ${ }^{47}$ (Supplementary Fig. 18). In addition to the morphological change, we observe a suppression of membrane fluctuations (Supplementary Fig. 19, Supplementary Movie 5), indicating a mechanical stabilization of the compartment $^{35,48}$ by the DNA-based exoskeleton mimic
(Supplementary Note 2). The stabilization effect could potentially be exploited for drug delivery applications. Both the morphological and the mechanical alterations are reversible (Supplementary Fig. 20): Addition of an acid led to $\mathrm{pH}$ decrease and hence to the detachment of the DNA origami from the GUV membrane. Notably, the GUVs relax back into their initial spherical shape (Fig. 4d, more images in Supplementary Figs. 21 and 22). Note that a $\mathrm{pH}$ decrease to $\mathrm{pH} 5.6$ is required to fully detach the DNA origami from the GUV membrane, which is below the $\mathrm{pH}$ decrease that can be provided by the E. coli (see Fig. 1). The larger $\mathrm{pH}$ gradients required for attachment and detachment of the DNA origami compared to the triplex strand alone can be explained with a cooperativity effect. Each DNA origami is modified with four triplexes. Therefore, complete detachment took several hours and hence the addition of an acid was necessary. The histograms in Fig. 4e quantify the $\mathrm{pH}$-reversible morphology change of the GUVs, revealing lower and more broadly distributed circularities when the DNA origami was attached at high $\mathrm{pH}$. Taken together, the self-assembly of nanoscopic pH-responsive building blocks could trigger the microscopic morphological remodeling of the shape of lipidmembrane-based synthetic cellular compartments. 
a

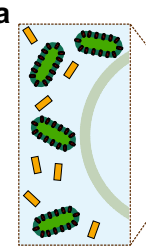

Step I - DNA origami attachment

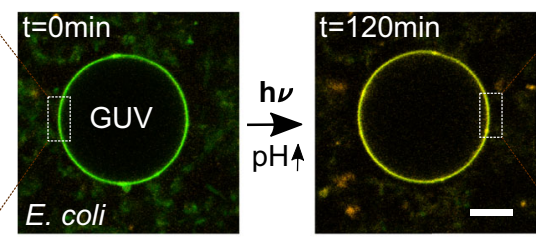

b

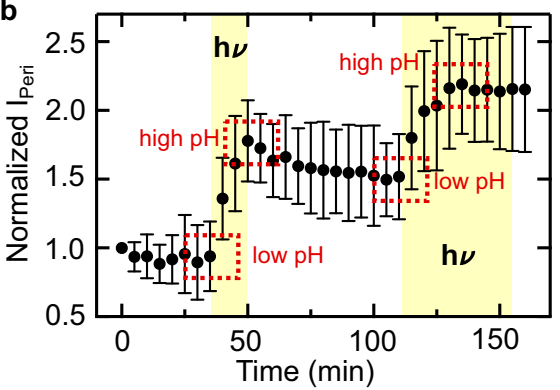

Step II - DNA origami polymerization

c
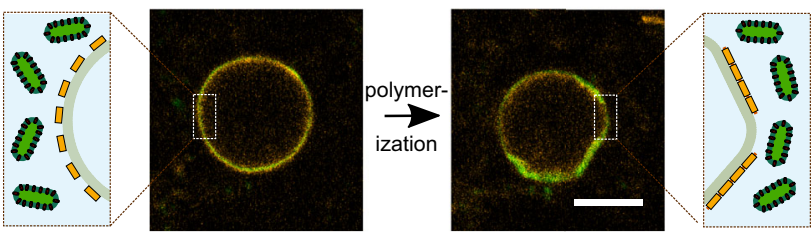

Fig. 5 Light-harvesting E. coli trigger DNA origami attachment and GUV deformation. a Confocal images of a GUV ( $\lambda_{e x}=488 \mathrm{~nm}$, green) functionalized with cholesterol-tagged DNA immersed in an E. coli and DNA origami ( $\lambda_{e x}=561 \mathrm{~nm}$, orange) containing solution before (left) and after (right) light illumination. Scale bar: $10 \mu \mathrm{m}$. After light illumination, the DNA origami (orange) attaches to the GUV due to the $\mathrm{pH}$ increase triggered by the E. coli. $\mathbf{b}$ Normalized fluorescence intensity $I_{\text {peri }}$ (mean \pm s.d., $n=11$ ) of the triplex-forming DNA at the GUV periphery monitored over time. The time period of illumination is indicated in yellow, illumination leads to a $\mathrm{pH}$ increase and hence DNA origami attachment. c Confocal images of a GUV $\left(\lambda_{\mathrm{ex}}=488 \mathrm{~nm}\right.$, green) after light-mediated DNA origami $\left(\lambda_{\mathrm{ex}}=561 \mathrm{~nm}\right.$, orange) attachment to the membrane and addition of the DNA staple strands at the scaffold seam which enable blunt-end stacking. DNA origami polymerization leads to the deformation of the GUV membrane within $2 \mathrm{~h}$. Scale bar: $10 \mu \mathrm{m}$. Source data is available for Fig. 5b.

Finally, we set out to combine the DNA origami-mediated $\mathrm{pH}$ sensitive deformation of GUVs with the light-responsive protonpumping capabilities of the E. coli. First, we showed that the GUVs remained stable in the E. coli culture and that we can attach the plain triplex-forming DNA to the GUV membrane upon illumination (Supplementary Figs. 23 and 24; Supplementary Movie 6). Thus, the $\mathrm{pH}$-signal-transduction between the topdown engineered $E$. coli and bottom-up assembled synthetic cells is also successful when the E. coli are used as external actuators. Next, we immersed the GUVs in a solution of E. coli and $\mathrm{pH}-$ sensitive DNA origami. We observed the attachment of the $\mathrm{pH}$ sensitive DNA origami to the GUV membrane upon illumination (Fig. 5a, Supplementary Movie 7). Fig. 5b quantifies the amount of DNA origami attachment, i.e., normalized fluorescence intensity at the GUV periphery $I_{\text {Peri }}$, over time during periods of illumination and darkness. DNA origami attachment happens repeatedly and only during periods of illumination, until saturation is reached. The attachment occurs due to the $\mathrm{pH}$ increase triggered by the light-harvesting E. coli. After illumination, in periods of darkness, the $\mathrm{pH}$ gradient dissipates. Nevertheless, the amount of DNA origami attachment remains roughly constant (Fig. 5b, $t=50 \mathrm{~min}$ until $t=110 \mathrm{~min}$ ). This can be attributed to the observed $\mathrm{pH}$ hysteresis effect (Supplementary Fig. 12): The $\mathrm{pH}$ would have to drop below the starting value for detachment, which cannot be achieved with the E. coli alone. Nevertheless, a second illumination cycle (from $t=110 \mathrm{~min}$ ) showed that the E. coli remain active and that the DNA origami attachment continued until saturation was reached.

After attachment, we enabled the polymerization of individual DNA origami monomers by adding the staple strands at the scaffold seam, which induce blunt-end stacking. This leads to the deformation of GUVs within two hours (Fig. 5c, Supplementary Movie 8), when the solution was illuminated with light, whereas GUVs remained spherical when they were left in the dark (Supplementary Figs. 25 and 26). Note that the deformation is weaker compared to the deformation achieved with conventional $\mathrm{pH}$ switching due to the smaller $\mathrm{pH}$ gradient. We can thus exploit the light-harvesting $E$. coli to actuate a morphology change of the GUVs.

\section{Discussion}

In summary, we have shown that the use of top-down engineered bacteria can enhance bottom-up assembled synthetic cells. The light-induced proton gradients we achieve with xenorhodopsinoverexpressing E. coli are not only larger than what was previously achieved with purified and reconstituted proteins - we also circumvent the laborious processes involved in their preparation. Especially membrane proteins, which can provide transient or chemically storable forms of energy as well as signal transduction and molecular transport in living cells, can be challenging to purify and reconstitute. Therefore, we can exploit the engineered $E$. coli to drive sophisticated downstream dynamics in synthetic cells. In particular, we demonstrate the $\mathrm{pH}$ sensitive attachment of a triplex-motif-carrying DNA origami to the compartment periphery upon illumination. The polymerized DNA origami, in turn, leads to a shape change of the GUVs triggered by the proton-pumping activity of the E. coli. The possibility to manipulate lipid membranes and not just the DNA nanostructures themselves broadens the scope of the popular DNA triplex-motif. For biotechnological applications, compartments that modify themselves as a response to environmental factors are highly desirable. More general, the integration of topdown engineered cells into bottom-up synthetic biology, contributing to bridge a long-standing divide, will provide the potential to realize diverse functions beyond light-harvesting ${ }^{49}$. We envision that the integration of top-down engineered components in synthetic cells will be a leap forward in their complexity and functionality.

\section{Methods}

Cloning. The plasmid pNR31 harboring the xenorhodopsin gene from Nanosalina (NsXeR) fused to the gene coding for superfolder-GFP (sf-GFP) was assembled by replacing the gene coding for proteorhodopsin in plasmid pNR $03^{7}$ with the NsXeR gene (Supplementary Table 1). Therefore, a codon-optimized NsXeR gene based on the amino-acid sequence ${ }^{25}$ with a $5^{\prime} \mathrm{NdeI}$ and a $3^{\prime} \mathrm{BamHI}$ restriction site was synthesized by GenScript (https://www.genscript.com) and cloned into the pUC57 plasmid. Using these two restriction enzymes (New England Biolabs, Ipswich, MA), the NsXeR gene was then subcloned into the pNR03 plasmid. The plasmid pNR33 harboring the NsXeR gene fused to mCherry (Supplementary Table 1) was assembled in multiple steps. First the sf-GFP gene in pNR03 was replaced by the gene coding for mCherry. To that end, the mCherry gene was amplified from the pNR09 plasmid using primers 5'-GGC GGA TCC ATG CAT AGC AAG GGC GAG-3' and 5'-GCC AAG CTT CTT GTA CAG C-3' (Microsynth AG) to introduce $5^{\prime}$ BamHI and $3^{\prime}$ HindIII restriction sites ${ }^{7}$. The resulting PCR-product was then cloned into plasmid pNR03 where it replaced the sf-GFP gene. Subsequently the same subcloning as for plasmid pNR31 was performed to replace the gene coding for proteorhodopsin with the NsXeR gene.

Overexpression of fusion-proteins in E. coli. E. coli C41 (DE3) cells (SigmaAldrich) were transformed with the plasmids pNR31 and pNR33. $100 \mathrm{~mL}$ Luria- 
Bertani (Fisher Scientific) liquid cultures ( $100 \mu \mathrm{g} / \mathrm{mL}$ ampicillin, Sigma-Aldrich) were inoculated 1:100 from overnight cultures. The E. coli cells were grown at $37^{\circ} \mathrm{C}$ while shaking at $220 \mathrm{rpm}$ until an $\mathrm{OD}_{600}$ of 0.4 was reached. Then, all-trans-retinal (Sigma-Aldrich) was added to a concentration of $10 \mu \mathrm{M}$ and the expression of the fusion-proteins was induced with the addition of $1 \mathrm{mM}$ isopropyl-D-

thiogalactopyranoside (IPTG, Sigma-Aldrich). The cells were incubated for another $4 \mathrm{~h}$ at $37^{\circ} \mathrm{C}$ while shaking at $220 \mathrm{rpm}$. Subsequently they were harvested by centrifugation $\left(3200 \times g\right.$ for $10 \mathrm{~min}$ at $\left.4^{\circ} \mathrm{C}\right)$ and resuspended in $150 \mathrm{mM} \mathrm{NaCl}$. The cells were stored at $4^{\circ} \mathrm{C}$ and protected from light until further use.

Photoactivity measurements with a micro $\mathrm{pH}$-electrode. E. coli cells overexpressing either XeR-GFP or XeR-mCherry were washed twice with $150 \mathrm{mM}$ $\mathrm{NaCl}\left(3200 \times \mathrm{g}\right.$ for $10 \mathrm{~min}$ at $\left.4^{\circ} \mathrm{C}\right)$ prior to photoactivity measurements. Immediately before the measurement, another washing step was performed. The bacteria were concentrated to an $\mathrm{OD}_{600}$ of 20. Photoactivity measurements were conducted using a micro $\mathrm{pH}$-electrode (InLab Micro Pro, Mettler Toledo, Columbus, $\mathrm{OH}$ ) and a sample volume of $800 \mu \mathrm{L}$. The $\mathrm{pH}$ was recorded every $10 \mathrm{~s}$. During the measurements the bacteria were protected from ambient light and continuously stirred to prevent sedimentation. The sample was illuminated with a KL 1500 LCD halogen lamp (Schott) for $5 \mathrm{~min}$ during each light-dark cycle. After each illumination-period the sample was kept in the dark for $10 \mathrm{~min}$. All measurements were performed at room temperature.

Confocal fluorescence microscopy. A confocal laser scanning microscope LSM 880, LSM 800 or LSM 700 (Carl Zeiss AG) was used for confocal imaging. The pinhole aperture was set to one Airy Unit and experiments were performed at room temperature. The images were acquired using a 20x objective (Plan-Apochromat 20x/0.8 M27, Carl Zeiss AG). Images were analyzed and processed with ImageJ (NIH, brightness and contrast adjusted) and Zen imaging software (Version 2.3). Confocal images were analyzed using a custom-written ImageJ macro (available here: https://doi.org/10.5281/zenodo.4738934). Cell-sized compartments were identified, their radius calculated and the intensity within the compartment center defined as mean inner intensity $\mathrm{I}_{\mathrm{In}}$. The peripheral intensity was determined by quantifying the maximum intensity along a line orthogonal to the compartment periphery. This was repeated every $18^{\circ}$ and the mean value taken as $I_{\text {Peri. }}$. The resulting data was plotted with Prism 8 (Version 8.4.3) and figures were compiled with Inkscape (Version 1.0rc1).

Formation of surfactant-stabilized water-in-oil droplets. Microfluidic PDMSbased (Sylgard 184, Dow Corning) devices for the formation of water-in-oil droplets were produced and assembled ${ }^{40}$. The device layouts of the single and twoinlet devices are shown in the Supplementary Fig. 7. The oil-phase contained $1.4 \mathrm{wt} \%$ of Perflouro-polyether-polyethylene glycol (PFPE-PEG) block-copolymer fluorosurfactants (PEG-based fluorosurfactant, Ran Biotechnologies, Inc.) dissolved in HFE-7500 oil (DuPont). The aqueous phase contained the encapsulated content and was varied as described in the corresponding sections. The fluid pressures to induce droplet formation were controlled by an Elveflow microfluidic flow control system or syringe pumps (Harvard Apparatus). The fluids for the syringe pumps were injected into the channels with $1 \mathrm{ml}$ syringes (Omnifix, B. Braun, Germany) connected by a cannula (Sterican ${ }^{\circledast} 0.4 \times 20 \mathrm{~mm}$, BL/LB, B.Braun) as well as PTFE-tubing $(0.4 \times 0.9 \mathrm{~mm}$, Bola) $)$ To observe the droplet production process, an Axio Vert.A1 (Carl Zeiss AG) inverse microscope was used. As an alternative to the microfluidic formation of droplets, the aqueous phase was layered on top of the oil phase within a microtube (Eppendorf) and droplet formation was induced by manual shaking 50 .

Photoactivity measurements in droplets. Photoactivity measurements in droplets were performed by encapsulating E. coli $\left(\mathrm{OD}_{600} \approx 20\right)$ with pyranine $(50 \mu \mathrm{M})$ into surfactant-stabilized droplets using the microfluidic device described above. The droplets were stored at $4^{\circ} \mathrm{C}$ after formation to allow for equilibration of the $\mathrm{pH}$ inside the droplet. Subsequently, droplets were sealed in an observation chamber and observed with confocal fluorescence microscopy. After 10 min of imaging in the dark, the sample was illuminated for 5 min using a Photonic PL 1000 lamp (light intensity $8 \mathrm{Mlx}$ using a $30 \mathrm{~W}$ halogen bulb). The lightguide was placed 5-10 $\mathrm{cm}$ above the sample. These cycles were repeated for $1 \mathrm{~h}$.

pH-sensitive attachment of DNA to the droplet periphery. Cholesterol-tagged DNA (sequence: 5' (Cy3)-ACCAGACAATACCACACAATTTT-CholTEG 3', HPLC purified) and the Cy-5 labeled triplex-forming DNA (sequence: 5' Cy5TTCTCTTCTCGTTTGCTCTTCTCTTGTGTGGTATTGTCTAAGAGAAGAG 3', adapted from Green et al. ${ }^{36}$, HPLC purified) were purchased from Biomers or Integrated DNA Technologies. Both DNA sequences were encapsulated in microfluidic droplets at a concentration of $1.5 \mu \mathrm{M}$ and $1 \mu \mathrm{M}$, respectively. For the calibration measurement (Fig. 3b), the aqueous solution inside the droplets additionally contained $50 \mathrm{mM}$ potassium phosphate buffer at the respective $\mathrm{pH}$. Propylamine (from Sigma Aldrich) and Trifluoracetic Acid (TFA, from Sigma Aldrich) were flushed to dynamically change the $\mathrm{pH}$ of the droplets' aqueous phase. For the co-encapsulation of the DNA together with the $E$. coli $\left(\mathrm{OD}_{600} \approx 20\right)$, a two-inlet droplet formation device was used (see Supplementary Fig. 7). Droplets were sealed in an observation chamber for confocal fluorescence imaging experiments.

GUVs electroformation and DNA attachment. GUVs consisting of $99 \%$ DOPC (1,2-dioleoyl-sn-glycero-3-phosphocholine, from Avanti Polar Lipids) and 1\% Atto488-DOPE (1,2-dioleoyl-sn-glycero-3-phosphoethanolamine-Atto488, from AttoTEC) in $120 \mathrm{mM}$ sucrose were produced via electroformation using a Vesicle Prep Pro (Nanion) ${ }^{34}$. An AC-current with an amplitude of $3 \mathrm{~V}$ and a frequency of $5 \mathrm{~Hz}$ was applied for $2 \mathrm{~h}$ at $37^{\circ} \mathrm{C}$. The cholesterol-tagged DNA and the triplexforming DNA were added to the GUVs at a concentration of $0.6 \mu \mathrm{M}$ and $0.4 \mu \mathrm{M}$, respectively, before the addition of the E. coli $\left(\mathrm{OD}_{600} \approx 20\right)$, in an unbuffered solution containing $150 \mathrm{mM} \mathrm{NaCl}$ and $5 \mathrm{mM} \mathrm{MgCl}_{2}$.

DNA origami design and assembly. DNA origami structures were adapted from an earlier design by Kopperger et al. ${ }^{51}$ using the open-access software cadnano ${ }^{52}$ Several changes were introduced, in particular: (1) Addition of nine DNA staple strand overhangs on the top layer, complementary to single stranded fluorescent Cy3-tagged DNA; (2) Addition of four single stranded overhangs on the bottom layer, complementary to the triplex-forming DNA; (3) Complete redesign of the edge staples resulting in a cross-shaped plate. The sticky cross DNA origami contained edge staples that finish the scaffold seam, enabling blunt-end stacking with neighboring origami. (4) Use of the longer single-stranded scaffold DNA, type p8064. A complete list of the DNA sequences is shown in Supplementary Data 1, the details of the design are shown in Supplementary Fig. 13. DNA origami was assembled by adding all unmodified staple strands (Integrated DNA Technologies, Inc., purification: standard desalting) in fivefold excess compared to the p8064 scaffold strand (tilibit nanosystems $\mathrm{GmbH}$ ). The solution contained $1 \times \mathrm{TAE}$ (Tris-Acetate-EDTA, Sigma-Aldrich) and $20 \mathrm{mM} \mathrm{MgCl}_{2}$ (Sigma-Aldrich) at $\mathrm{pH}$ 7.4. The DNA origami was annealed in a thermocycler (Bio-Rad T100) that controls a temperature ramp from $70^{\circ} \mathrm{C}$ to $20^{\circ} \mathrm{C}$ over $12 \mathrm{~h}$ and successively holds the temperature at $40^{\circ} \mathrm{C}$ for at least $3 \mathrm{~h}^{51}$. The unpurified samples were stored at $4{ }^{\circ} \mathrm{C}$ until further use.

Purification of the DNA origami. Prior to purification from excess staples, the DNA origami was mixed with $1 \mu \mathrm{M}$ Cy3-tagged single-stranded DNA (Integrated DNA Technologies, Inc., DNA sequence: 5' Cy3- AAAAAAAAAAAAAAAAAA AA 3', purification: HPLC) as well as a pH-sensitive triplex-forming DNA motif (Integrated DNA Technologies, Inc., DNA sequence: $5^{\prime}$ TTCTCTTCTCGTTTG CTCTTCTCTTGTGTGGTATTGTCTAAGAGAAGAGTTTGATGCATAGAA GG 3). The DNA origami was then suspendend in $500 \mu \mathrm{L}$ of $1 \times \mathrm{TAE}, 5 \mathrm{mM}$ $\mathrm{MgCl}_{2}$ and purification was performed by spin filtration in a Biofuge Fresco microlitre centrifuge (Heraeus 75005521) using $100 \mathrm{kDa}$ cutoff filters from Amicon (Amicon Ultra-15, PLHK Ultracel-PL Membran, UFC910008) ${ }^{31}$. After filtration, the $\mathrm{MgCl}_{2}$ concentration was raised to $20 \mathrm{mM}$ again. Before the experiment, microvolume spectrophotometry (PEQLAB Biotechnologie $\mathrm{GmbH}$ ) yielded a DNA origami concentration of $6.54 \pm 0.42 \mathrm{nM}$.

Cryo electron microscopy. In total, $3 \mu \mathrm{L}$ of the assembled DNA origami in $10 \mathrm{mM}$ sodium phosphate $\mathrm{pH} 8.3$ containing $20 \mathrm{mM} \mathrm{MgCl}$ were blotted for $5-10 \mathrm{~s}$ in a (Vitrobot Mark IV, Thermo Fischer) on Quantifoli 2/1 grids with zero blot force at $100 \%$ humidity. Plunge frozen samples were imaged in a Krios equipped with a K3 camera behind an energy filter at a pixel size of $0.137 \mathrm{~nm}$. Images were taken by single particle program (EPU, Thermo Fischer) with a a total dose of 20 e/A2. Movies of 20 frames were corrected ${ }^{53}$ then cropped, normalized, low-pass filtered $(0.0625)$ and $4 \mathrm{x}$ binned ${ }^{54}$.

GUV deformation with pH-sensitive DNA origami. The DNA origami (in $1 \mathrm{x}$ TAE, $20 \mathrm{mM} \mathrm{MgCl} 2$ ) was incubated with cholesterol-tagged DNA at $50 \mathrm{nM}$ for 25 minutes and immediately mixed with Atto488-labeled iso-osomotic (120 mOsmol) GUVs in a ratio of one to three. DNA origami-coated GUVs were imaged after 24 hours of incubation in the fridge. Subsequently, the GUVs were incubated for another 24 hours with $48 \mathrm{mM} \mathrm{KH}_{2} \mathrm{PO}_{4}$ buffer in order to detach the DNA origami from the GUV membrane.

GUV deformation with pH-sensitive DNA origami and light-responsive $E$. coli. For these experiments, the DNA origami was suspended in a solution of $75 \mathrm{mM}$ $\mathrm{NaCl}$ and $10 \mathrm{mM} \mathrm{MgCl}_{2}$ without addition of a $\mathrm{pH}$-buffering agent. In order to prevent DNA origami polymerization prior to GUV-attachment, the staple strands at the scaffold seam were excluded. The single-stranded scaffold loops prevent base stacking. Subsequently, Atto488-labeled GUVs in sucrose (195 mOsmol) were diluted in $75 \mathrm{mM} \mathrm{NaCl}$ and $10 \mathrm{mM} \mathrm{MgCl}_{2}$ and mixed with $2 \mu \mathrm{M}$ cholesterol-tagged DNA. After $5 \mathrm{~min}$ incubation, E. coli resuspended at an $\mathrm{OD}_{600}=60$ in $75 \mathrm{mM}$ $\mathrm{NaCl}$ and $10 \mathrm{mM} \mathrm{MgCl}_{2}$ and DNA origami were mixed in a 1:1:1 ratio with GUVs. For the DNA origami attachment the solution was put into an observation chamber and illuminated with white light during confocal imaging. For subsequent GUV deformation, the staples at the scaffold seam were added to induce base stacking interactions between the membrane-bound DNA origami. The solution 
was illuminated for $30 \mathrm{~min}$ in bulk before the addition of $50 \mathrm{nM}$ staple strands and imaged $14 \mathrm{~h}$ later.

Statistics and reproducibility. The experiments were performed independently at least two times. In particular, values in Fig. $1 \mathrm{~b}$ correspond to three independent experiments and in Fig. 1d to four independent experiments. Experiments for Figs. 2e, $3 \mathrm{~b}, \mathrm{~d}$ and $4 \mathrm{e}$ were performed two times or more. Fig. 5 was only replicated once, however due to the sequential attachment via light and appropriate controls, we believe that this is adequate. All representative confocal, atomic force and electron microscopy images are only a subset of at least 10 or more images showing similar results.

\section{Data availability}

The data that support the findings of this study are available from the corresponding author upon reasonable request. Source data for main figures $1 b, c, d, 2 e, 3 b, d, 4 e, 5 b$ and supplementary figures 1a, c, d, 2, 3a, b, 5, 8, 12, 16, 18b, 19a, b and 24c are provided with this paper.

\section{Code availability}

Image $\mathrm{J}$ macro for the analysis of the intensity inside the compartment and at the compartment periphery is provided under the following link: https://doi.org/10.5281/ zenodo.4738934.

Received: 28 January 2021; Accepted: 27 May 2021;

Published online: 25 June 2021

\section{References}

1. Benner, S. A. \& Sismour, A. M. Synthetic biology. Nat. Rev. Genet. 6, 533-543 (2005).

2. Schwille, P. Jump-starting life? Fundamental aspects of synthetic biology. J. Cell Biol. 210, 687-690 (2015).

3. Cameron, D. E., Bashor, C. J. \& Collins, J. J. A brief history of synthetic biology. Nat. Rev. Microbiol. 12, 381-390 (2014).

4. Zhang, F., Carothers, J. M. \& Keasling, J. D. Design of a dynamic sensorregulator system for production of chemicals and fuels derived from fatty acids. Nat. Biotechnol. 30, 354-359 (2012).

5. Anderson, J., Clarke, E., Arkin, A. \& Voigt, C. Environmentally Controlled Invasion of Cancer Cells by Engineered Bacteria. J. Mol. Biol. 355, 619-27 (2006).

6. Schuergers, N., Werlang, C. \& Boghossian, A. A. A synthetic biology approach to engineering living photovoltaics. Energy Environ. Sci. 10, 1102-1115 (2017).

7. Ritzmann, N. et al. Fusion domains guide the oriented insertion of lightdriven proton pumps into liposomes. Biophys. J. 113, 1181-1186 (2017).

8. Ausländer, S., Ausländer, D. \& Fussenegger, M. Synthetic biology-the synthesis of biology. Angewandte Chemie International Edition 56, 6396-6419 (2017).

9. Göpfrich, K., Platzman, I. \& Spatz, J. P. Mastering complexity: towards bottom-up construction of multifunctional eukaryotic synthetic cells. Trends Biotechnol. 36, 938-951 (2018).

10. Schwille, P. et al. MaxSynBio: avenues towards creating cells from the bottom up. Angewandte Chemie Int. Ed. 57, 13382-13392 (2018).

11. Supramaniam, P., Ces, O. \& Salehi-Reyhani, A. Microfluidics for artificial life: techniques for bottom-up synthetic biology. Micromachines 10, 299 (2019).

12. Otrin, L. et al. Toward artificial mitochondrion: mimicking oxidative phosphorylation in polymer and hybrid membranes. Nano Lett. 17 6816-6821 (2017).

13. Berhanu, S., Ueda, T. \& Kuruma, Y. Artificial photosynthetic cell producing energy for protein synthesis. Nat. Commun. 10, 1325 (2019).

14. Miller, T. E. et al. Light-powered $\mathrm{CO}_{2}$ fixation in a chloroplast mimic with natural and synthetic parts. Science 368, 649-654 (2020).

15. Keber, F. C. et al. Topology and dynamics of active nematic vesicles. Science 345, 1135-1139 (2014).

16. Bartelt, S. M., Steinkühler, J., Dimova, R. \& Wegner, S. V. Light-Guided Motility of a Minimal Synthetic Cell. Nano Lett. 18, 7268-7274 (2018)

17. Jahnke, K. et al. Engineering light-responsive contractile actomyosin networks with DNA nanotechnology. Adv. Biosyst. 2020, 2000102.

18. Steinkühler, J. et al. Controlled division of cell-sized vesicles by low densities of membrane-bound proteins. Nat. Commun. 11, 1-11 (2020).

19. Schwarz-Schilling, M., Aufinger, L., Mückl, A. \& Simmel, F. C. Chemical communication between bacteria and cell-free gene expression systems within linear chains of emulsion droplets. Integrative Biol. 8, 564-570 (2016).

20. Lentini, R. et al. Two-way chemical communication between artificial and natural cells. ACS Central Sci. 3, 117-123 (2017).
21. Lentini, R. et al. Integrating artificial with natural cells to translate chemical messages that direct E. coli behaviour. Nat. Commun. 5, 1-6 (2014).

22. Staufer, O.. Schröter, M., Platzman, I. \& Spatz, J. P. Bottom-up assembly of functional intracellular synthetic organelles by droplet-based microfluidics. Small 2020, 1906424.

23. Mehta, A. P. et al. Engineering yeast endosymbionts as a step toward the evolution of mitochondria. Proc. Natl. Acad. Sci. USA 115, 11796-11801 (2018).

24. Lau, Y. H., Giessen, T. W., Altenburg, W. J. \& Silver, P. A. Prokaryotic nanocompartments form synthetic organelles in a eukaryote. Nat. Commun. 2018, 9.

25. Shevchenko, V. et al. Inward H+ pump xenorhodopsin: mechanism and alternative optogenetic approach. Sci. Adv. 3, e1603187 (2017).

26. Inoue, $\mathrm{K}$. et al. A natural light-driven inward proton pump. Nat. Commun. 7, 13415 (2016)

27. Marušič, N. et al. Constructing artificial respiratory chain in polymer compartments: Insights into the interplay betweenbo3oxidase and the membrane. Proc. Natl. Acad. Sci. 117, 15006-15017 (2020).

28. Goers, R. et al. Optimized reconstitution of membrane proteins into synthetic membranes. Commun. Chem. 2018, 1.

29. Kano, K. \& Fendler, J. H. Pyranine as a sensitive $\mathrm{pH}$ probe for liposome interiors and surfaces. $\mathrm{pH}$ gradients across phospholipid vesicles. Biochimica et Biophysica Acta (BBA) - Biomembranes 509, 289-299 (1978).

30. Langecker, M. et al. Synthetic lipid membrane channels formed by designed DNA nanostructures. Science 338, 932-6 (2012).

31. Göpfrich, K. et al. Large-conductance transmembrane porin made from DNA origami. ACS Nano 2016, 10.

32. Czogalla, A. et al. Amphipathic DNA origami nanoparticles to scaffold and deform lipid membrane vesicles. Angewandte Chemie International Edition 54, 6501-5 (2015).

33. Franquelim, H. G., Khmelinskaia, A., Sobczak, J.-P., Dietz, H. \& Schwille, P. Membrane sculpting by curved DNA origami scaffolds. Nat. Commun. 9, 81 (2018).

34. Göpfrich, K. et al. DNA-tile structures lead to ionic currents through lipid membranes. Nano Lett. 15, 3134-3138 (2015).

35. Franquelim, H. G., Dietz, H. \& Schwille, P. Reversible membrane deformations by straight DNA origami filaments. Soft Matter 17, 276 (2020).

36. Green, L., Amodio, A., Subramanian, H. K. K., Ricci, F. \& Franco, E. pHdriven reversible self-assembly of micron-scale DNA scaffolds. Nano Lett. 17, 7283-7288 (2017)

37. Göpfrich, K., Urban, M. J., Frey, C., Platzman, I. \& Spatz, J. P. Dynamic actuation of DNA-assembled plasmonic nanostructures in microfluidic cellsized compartments. Nano Lett. 20, 1571-1577 (2020)

38. Kuzyk, A., Urban, M. J., Idili, A., Ricci, F. \& Liu, N. Selective control of reconfigurable chiral plasmonic metamolecules. Sci. Adv. 3, e1602803 (2017).

39. Porchetta, A., Idili, A., Vallée-Bélisle, A. \& Ricci, F. General strategy to introduce $\mathrm{pH}$-induced allostery in DNA-based receptors to achieve controlled release of ligands. Nano Lett. 15, 4467-4471 (2015).

40. Jahnke, K. et al. Programmable functionalization of surfactant-stabilized microfluidic droplets via DNA-tags. Adv. Funct. Mater. 29, 1808647 (2019).

41. Jahnke, K., Grubmüller, H., Igaev, M. \& Göpfrich, K. Choice of fluorophore affects dynamic DNA nanostructures. Nucleic Acids Res. 2021, gkab201.

42. Jia, H.-R., Zhu, Y.-X., Chen, Z. \& Wu, F.-G. Cholesterol-assisted bacterial cell surface engineering for photodynamic inactivation of gram-positive and gram-negative bacteria. ACS Appl. Mater. Interfaces 9, 15943-15951 (2017).

43. Cooper, M. E., Gregory, S., Adie, E. \& Kalinka, S. pH-Sensitive Cyanine Dyes for Biological Applications. J. Fluorescence 12, 425-429 (2002).

44. Kilchherr, F. et al. Single-molecule dissection of stacking forces in DNA Science 353, aaf5508 (2016).

45. Vogel, S. K. et al. Symmetry breaking and emergence of directional flows in minimal actomyosin cortices. Cells 9, 1432 (2020).

46. Kocabey, S. et al. Membrane-assisted growth of DNA origami nanostructure arrays. ACS Nano 9, 3530-3539 (2015).

47. Schaich, M., Sobota, D., Sleath, H., Cama, J. \& Keyser, U. F. Characterization of lipid composition and diffusivity in OLA generated vesicles. Biochimica et Biophysica Acta (BBA) - Biomembranes 1862, 183359 (2020).

48. Baumann, K. N. et al. Coating and stabilization of liposomes by clathrininspired DNA self-assembly. ACS Nano 14, 2316-2323 (2020).

49. Elani, Y. Interfacing living and synthetic cells as an emerging frontier in synthetic biology. Angewandte Chemie Int. Edi. 59, 2-12 (2020).

50. Göpfrich, K. et al. One-pot assembly of complex giant unilamellar vesiclebased synthetic cells. ACS Synthetic Biology 8, 937-947 (2019).

51. Kopperger, E. et al. A self-assembled nanoscale robotic arm controlled by electric fields. Science 359, 296-301 (2018).

52. Douglas, S. M. et al. Rapid prototyping of 3D DNA-origami shapes with caDNAno. Nucleic Acids Res. 37, 5001-5006 (2009). 
53. Zheng, S. Q. et al. MotionCor2: anisotropic correction of beam-induced motion for improved cryo-electron microscopy. Nat. Methods 14, 331-332 (2017).

54. Schindelin, J. et al. Fiji: an open-source platform for biological-image analysis. Nat. Methods 9, 676-682 (2012).

\section{Acknowledgements}

K.J., R.R.S., J.P.S. and K.G. acknowledge funding from the Deutsche For schungsgemeinschaft (DFG, German Research Foundation) under Germany's Excellence Strategy via the Excellence Cluster 3D Matter Made to Order (EXC-2082/1 - 390761711). K.J. thanks the Carl Zeiss Foundation. J.P.S and I.P. acknowledge funding from the European Research Council, Grant Agreement no. 294852, SynAd, the MaxSynBio Consortium (jointly funded by the Federal Ministry of Education and Research of Germany and the Max Planck Society), from the SFB 1129 of the German Science Foundation and from the VolkswagenStiftung (priority call 'Life?'). J.P.S. is the Weston Visiting Professor at the Weizmann Institute of Science. G.H. and R.R.S. acknowledge the services SDShd and bwHPC supported by the Ministry of Science, Research and the Arts Baden-Württemberg. The Krios microscope is part of the Cluster of Excellence "CellNetworks" (Exc 81) at the Universität Heidelberg. K.G. received funding from the European Union Horizon 2020 research and innovation program under the Marie Skłodowska-Curie grant agreement No. 792270. The authors acknowledge the Max Planck Society for its general support. N.R. and D.J.M. thank the Swiss Nanoscience Institute (SNI, Basel, Switzerland), the Swiss National Science Foundation and NCCR Molecular Systems Engineering for their support.

\section{Author contributions}

K.J. performed most experiments and analysis. N.R. designed and prepared genetically engineered E. coli and performed $\mathrm{pH}$ electrode measurements. J.F. and K.G. designed the DNA origami. J.F. and K.J. carried out $\mathrm{pH}$-sensitive deformation experiments. A.N. and K.J. established the use of pyranine as pH-sensor within droplets or GUVs. Y.D. and K.J. performed and analyzed $\mathrm{pH}$-sensitive DNA attachment to GUVs. T.A. helped in analyzing the fluorescence intensity ratios. G.H. and R.R.S. designed and carried out cryoEM experiments. K.J., K.G., I.P., D.J.M. and J.P.S. designed the study. K.J. and K.G. wrote the manuscript with help from all authors.

\section{Funding}

Open Access funding enabled and organized by Projekt DEAL.

\section{Competing interests}

The authors declare no competing interests.

\section{Additional information}

Supplementary information The online version contains supplementary material available at https://doi.org/10.1038/s41467-021-24103-x.

Correspondence and requests for materials should be addressed to K.G

Peer review information Nature Communications thanks the anonymous reviewers for their contribution to the peer review of this work.

Reprints and permission information is available at http://www.nature.com/reprints

Publisher's note Springer Nature remains neutral with regard to jurisdictional claims in published maps and institutional affiliations.

cc (i) Open Access This article is licensed under a Creative Commons Attribution 4.0 International License, which permits use, sharing, adaptation, distribution and reproduction in any medium or format, as long as you give appropriate credit to the original author(s) and the source, provide a link to the Creative Commons license, and indicate if changes were made. The images or other third party material in this article are included in the article's Creative Commons license, unless indicated otherwise in a credit line to the material. If material is not included in the article's Creative Commons license and your intended use is not permitted by statutory regulation or exceeds the permitted use, you will need to obtain permission directly from the copyright holder. To view a copy of this license, visit http://creativecommons.org/ licenses/by/4.0/.

(C) The Author(s) 2021 